\title{
Anticorpos Contra Beta2-Glicoproteína I como Fatores de Risco para Infarto Agudo do Miocárdio
}

\author{
Aline Ranzolin, Jussara Marilú Bohn, Gary L. Norman, Euler Manenti, Luis Carlos Bodanese, \\ Carlos Alberto von Mühlen, Henrique Luiz Staub \\ Porto Alegre, RS
}

\section{Objetivo}

Determinar se títulos elevados de anticorpos contra o cofator fosfolipídico beta2-glicoproteína I (beta2-gpl) se associam a risco aumentado de infarto agudo do miocárdio.

\section{Métodos}

Incluídos 82 pacientes com infarto agudo do miocárdio e 82 controles, avaliados quanto à idade, sexo, raça, hipertensão, tabagismo, cardiopatia prévia, história de diabetes mellitus e hipercolesterolemia. Anticorpos anticardiolipina e antibeta2-gpl IgA, IgG e IgM foram detectados por imunoensaio. Odds ratios (OR) ajustados para fatores de risco foram obtidos através de regressão logística.

\section{Resultados}

A média de idade para casos e controles foi, respectivamente, de 57,7 e 51,1 anos ( $P=0,003)$, predominando homens $(P=0.005)$ e a raça branca em ambos os grupos $(P=0.798)$. Entre os fatores de risco, história de diabetes (OR 5,3; IC95\% 1,9 a 14,9; $P=0,001)$ e cardiopatia prévia (OR 4,7; IC95\% 2,0 a 10,7; $P<0,001$ ) foram as associações mais consistentes com o infarto do miocárdio. A freqüência de anticorpos anticardiolipina IgG, IgM e IgA não diferiu em casos e controles $(P=1,000)$. Anticorpos IgA contra beta2-gpl IgA foram mais freqüentes em casos do que em controles $(P=0.054)$. O OR ajustado para anticorpos IgA anti-beta2-gp/ IgA foi 3,4 (IC95\% 1,3 a 9,1; $P=0,015)$.

\section{Conclusão}

Anticorpos IgA antibeta2-gpl, mas não anticardiolipina, parecem se comportar como fatores de risco independentes para o infarto, o que pode representar um elo entre autoimunidade e aterosclerose em pacientes com infarto agudo do miocárdio.

\section{Palavras-chave}

anticorpos antifosfolípides, anticorpos antibeta2-glicoproteína I, infarto agudo do miocárdio

Hospiral São Lucas da PUCRS

Endereço para Correspondência: Dr. Henrique Luiz Staub

Av. Ipiranga, 6690 - Sala 220 - Reumatologia - Cep 90610-000

Porto Alegre, RS - E-mail: henriquestaub@terra.com.br

Recebido para Publicação em 30/5/03

Aceito em 6/1/04
Fatores imunológicos podem estar envolvidos na etiopatogênese da aterosclerose. 0 papel dos anticorpos contra fosfolípides (FL) ou contra cofatores fosfolipídicos no processo aterosclerótico ainda não está claro ${ }^{1}$.

Anticorpos antifosfolípides, sejam anticorpos anticardiolipina (aCL) ou o anticoagulante lúpico, estão relacionados à sindrome antifosfolipídica, que é caracterizada por tromboses arterial e venosa e morbidade gestacional, e, atualmente considerada a causa mais comum de hipercoagulabilidade adquirida em adultos jovens ${ }^{2}$.

O infarto agudo do miocárdio ocorre em 4-20\% dos pacientes com síndrome antifosfolipídica ${ }^{3}$. Em uma coorte recente de 1000 pacientes com síndrome antifosfolipídica, o infarto agudo do miocárdio foi observado em $2,8 \%$ dos casos $^{4}$.

O cofator fosfolipídico beta2-glicoproteína I (beta2-gpl) é um anticoagulante natural ${ }^{5}$. De grande interesse, este cofator foi encontrado em placas ateroscleróticas ${ }^{6}$, havendo relatos de indução de aterosclerose em camundongos deficientes de receptor-LDL imunizados com beta2-gpl 7 .

Anticorpos antibeta2-gpl por imunoensaio foram descritos em casos de síndrome antifosfolipídica definida ${ }^{8}$, mas também em pacientes com hipertensão pulmonar tromboembólica ${ }^{9}$, infarto cerebral $^{10}$ e doença coronariana ${ }^{11}$.

A freqüência dos anticorpos anticardiolipina e antibeta2-gpl, bem como seu papel em pacientes com infarto agudo do miocárdio, têm sido matéria de discussão. Nosso estudo inclui um perfil completo de anticorpos anticardiolipina e antibeta2-gpl em coronariopatas agudos, analisa sua freqüência em pacientes com infarto agudo do miocárdio e aventa a possibilidade de que anticorpos anticardiolipina e antibeta2-gpl funcionem como fatores de risco independentes para infarto agudo do miocárdio.

\section{Métodos}

Avaliamos os anticorpos anticardiolipina e anti-beta2-gpl em pacientes com infarto agudo do miocárdio e em controles arrolados em um estudo de caso-controle. Somente casos incidentes foram avaliados.

O diagnóstico do infarto foi efetuado por cardiologistas de acordo com algoritmos previamente relatados, incluindo a história clínica, alterações eletrocardiográficas seriadas e testes laboratoriais confirmatórios de necrose miocárdica ${ }^{12}$ e permanecendo o cardiologista cego para os resultados de quaisquer anticorpos em questão.

Os casos compreenderam pacientes com mais de 16 anos, admitidos no hospital nos primeiros 7 dias de início dos sintomas. Os pacientes não foram selecionados por sexo ou raça. 0 próprio 
paciente ou procurador provieram o consentimento informado. Auto-identificação determinou a raça/etnia.

Os critérios de exclusão foram: a) endocardite infecciosa; b) neoplasias (atual ou passada); c) infecção pelo vírus da imunodeficiência humana ou treponema pallidum; d) presença de causas hereditárias conhecidas para trombose como homocistinúria ou mutação do fator V (Leiden); e) diagnóstico prévio de síndrome antifosfolipídica ou outra doença do tecido conjuntivo.

O grupo controle foi constituído de pacientes sem infarto agudo do miocárdio admitidos em enfermarias de Ortopedia devido a fraturas ou desordens músculo-ligamentares. Os critérios de exclusão foram: a) osteonecrose; b) infecções, neoplasias, doenças hereditárias, síndrome antifosfolipídica ou doença do tecido conjuntivo, conforme listados acima para "casos".

Informações históricas, demográficas e clínicas foram obtidas por revisão de prontuário e entrevista com o paciente e sua família. Os fatores de risco incluídos para infarto foram: 1) idade, sexo, raça/etnia; 2) história de hipertensão (diagnóstico confirmado quando as pressões sistólica ou diastólica > que 160 ou $95 \mathrm{mmHg}$, respectivamente, ou quando o paciente estivesse em uso de medicação para hipertensão) ${ }^{12}$; 3) tabagismo, de acordo com os critérios do Conselho Inglês de Pesquisa Médica; 4) história de doença cardíaca (fibrilação atrial ou doença arterial coronariana, definida como infarto miocárdico prévio, angina ou procedimento de revascularização); 5) história de diabetes mellitus, conforme história médica ou uso de insulina ou hipoglicemiante oral; 6) hipercolesterolemia, baseada em colesterol total $>200 \mathrm{mg} / \mathrm{dl}$, LDL-colesterol > $130 \mathrm{mg} / \mathrm{dl}$ ou índice colesterol total/HDLcolesterol $>$ de $5^{13}$.

Amostras de soro foram centrifugadas e congeladas em, no máximo, $2 \mathrm{~h}$ após a coleta e guardadas $\mathrm{a}-70^{\circ} \mathrm{C}$ até a testagem laboratorial em ELISA (enzyme-linked immunoabsorbant assays).

Anticorpos anticardiolipina IgG/lgM/lgA ELISA (INOVA Quantalite cardiolipin kits, INOVA Diagnostics, Inc., San Diego, USA) foram detectados de acordo com descrição prévia. Os resultados para os isotipos IgG e IgM foram relatados em unidades fosfolipídicas IgG (GPL) e unidades fosfolipídicas IgM (MPL), onde 1 unidade é igual a $1 \mathrm{mg} / \mathrm{ml}$ de IgG ou lgM. Somente amostras com níveis moderados a altos de anticorpos anticardiolipina IgG ou IgM (acima de $20 \mathrm{GPL}$ ou $20 \mathrm{MPL}$ ) foram consideradas como positivas em nosso estudo. Títulos de anticorpos anticardiolipina IgA foram considerados positivos quando acima de 15 unidades $^{14}$.

Anticorpos antibeta2-gpl IgA, IgG e IgM foram detectados de acordo com relato prévio (INOVA Quantalite beta2-gpl kits, INOVA Diagnostics, Inc., San Diego, USA). Brevemente, $50 \mu$ l de beta2gpl humana purificada (concentração $10 \mu \mathrm{g} / \mathrm{ml}$ ) foram acopladas a orifícios de placas de poliestireno. Controles pré-diluídos e soro diluído de pacientes (1/100) foram adicionados a orifícios separados, permitindo que qualquer anticorpo antibeta2-gpl presente se ligasse ao antígeno imobilizado. Amostras não-ligadas ao antígeno foram lavadas. Anticorpos anti-lgG, anti-lgM ou anti-lgA (100 $\mu$ l) humanos ligados a peroxidase foram adicionados aos orifícios. Uma segunda incubação permitiu aos anticorpos anti-humanos ligarem-se a qualquer anticorpo de paciente que tivesse aderido à placa. Após a lavagem de quaisquer anticorpos anti-humano não-ligados, a atividade enzimática remanescente foi medida pela adição de um substrato cromogênico. $O$ ensaio foi avaliado por medidas espec- paciente foi comparada com a dos controles. Títulos foram considerados positivos quando acima de 20 unidades para anticorpos IgG, IgM ou IgA anti-beta2-gp ${ }^{15}$.

Odds ratios com intervalo de confiança de 95\% (IC95\%I) foram calculados através de regressão logística, com ajuste para idade, sexo, raça, história de hipertensão, tabagismo, doença cardíaca prévia, história de diabetes e hipercolesterolemia. Todas as interações de primeira ordem entre fatores de risco conhecidos para infarto agudo do miocárdio e níveis de anticorpos foram examinadas. A escala de Hopkins para $\mathrm{OR}^{16}$ foi utilizada, onde um OR entre 1-1,5 foi considerado trivial; entre 1,5-3,5 como pequeno; entre 3,5-9,0 como moderado; entre 9,0-32 como forte; e acima de 32 como muito forte. 0 teste de Wald ${ }^{17}$ foi usado para avaliar a significância do OR ajustado por regressão logística. Os testes exato de Fisher e do qui-quadrado foram usados para comparação de variáveis categóricas, e o teste $t$ de Student usado para comparação de variáveis contínuas; um nível de significância de 5\% $(P<0.05)$ foi adotado. Todas as análises foram obtidas através do uso do SPSS para Windows, versão 8.0, Chicago, IL.

\section{Resultados}

Foram incluídos em nosso estudo 82 pacientes com infarto agudo do miocárdio e 82 controles. As características clínicas e demográficas de casos e controles se encontram na tabela I. Pacientes com infarto agudo do miocárdio eram, predominantemente, homens e mais velhos $(P=0,003)(P=0,005)$ e determinaram pequeno risco (OR 2,5; IC95\% 1,3 a 4,7). A raça branca predominou em casos e controles.

As informações sobre os fatores de risco para casos e controles são apresentadas na tabela II, sendo que os fatores de risco conhecidos para infarto agudo do miocárdio foram mais freqüentes em casos do que em controles. História de diabetes (OR 5,3; IC95\% 1,9 a 14,9; P=0,001) e cardiopatia prévia (OR 4,7; IC95\% $2,0$ a 10,$7 ; \mathrm{P}<0,001)$ compreenderam as associações mais consistentes com infarto agudo do miocárdio.

A tabela III categoriza casos e controles pelos níveis de anticorpos anticardiolipina e antibeta2-gpl. A freqüência de IgA antibeta2-gpl, mas não a de outros anticorpos, foi maior em casos do que em controles $(P=0,054)$.

Os OR ajustado para fatores de risco (idade, sexo, raça, história de hipertensão, tabagiasmo, doença cardíaca prévia, história de DM e hipercolesterolemia) acham-se na tabela IV.

Teste positivo para o anticorpo IgG antibeta2-gpl IgG forneceu um OR de 0,1 (IC95\% zero a 1,0); o valor $\mathrm{P}$ ajustado no teste de Wald foi limítrofe para umo papel protetor para este anticorpo $(P=0,055)$. A ocorrência do anticorpo IgA antibeta2-gpl IgA determinou um risco moderado para infarto agudo do miocárdio (OR ajustado 3,4; IC95\%. 1,3 a 9,1; $P=0,015)$.

\section{Discussão}

Este estudo de caso-controle de casos incidentes incluiu um perfil completo de anticorpos anticardiolipina e antibeta2-gpl em população não-selecionada de adultos com infarto agudo do miocárdio.

A média de idade dos casos diferiu significantemente dos controles. Homens predominaram nos casos. Idade e sexo, bem como outros fatores de risco, foram ajustados por regressão logística. Entre os fatores de risco conhecidos, história de diabetes 
Anticorpos contra Beta2-glicoproteína I como fatores de risco para IAM

\begin{tabular}{|c|c|c|c|c|}
\hline & Casos $(n=82)$ & Controles $(n=82)$ & $P$ & OR $($ IC95\%)\# \\
\hline Média de idade (DP\#\#) & $57,7(10,4)$ & $51,1(17)$ & $0,003^{*}$ & \\
\hline Homens & $55(67,1 \%)$ & $37(45,1 \%)$ & $0,005^{\star *}$ & $2,5(1,3-4,7)$ \\
\hline Raça branca & $74(90,2 \%)$ & $73(89 \%)$ & $0,798 * *$ & $1,1(0,4-3,1)$ \\
\hline
\end{tabular}

\begin{tabular}{|c|c|c|c|c|}
\hline & $\begin{array}{l}\text { Casos } \\
(n=82)\end{array}$ & $\begin{array}{c}\text { Controles } \\
(n=82)\end{array}$ & $P^{*}$ & OR (IC95\%)\# \\
\hline \multicolumn{5}{|l|}{ Fatores de risco } \\
\hline História de hipertensão & $46(56,1 \%)$ & $22(26,8 \%)$ & $<0,001$ & $3,5(1,8-6,7)$ \\
\hline Tabagismo & $46(56,1 \%)$ & $28(34,1 \%)$ & 0,005 & $2,5(1,3-4,6)$ \\
\hline Doença cardíaca prévia & $30(36,6 \%)$ & $9(11 \%)$ & $<0,001$ & $4,7(2,0-10,7$ \\
\hline História de diabetes mellitus & $21(25,6 \%)$ & $5(6,1 \%)$ & 0,001 & $5,3(1,9-14,9$ \\
\hline Hipercolesterolemia & $34(41,5 \%)$ & $16(19,5 \%)$ & 0,002 & $2,9(1,4-5,9)$ \\
\hline
\end{tabular}

\begin{tabular}{|c|c|c|c|}
\hline \multicolumn{4}{|c|}{$\begin{array}{l}\text { Tabela III - Freqüência de anticorpos anticardiolipina e antibeta2-gpI } \\
\text { para casos e controles }\end{array}$} \\
\hline & Casos $(n=82)$ & Controles $(n=82)$ & $\mathrm{P}$ \\
\hline aCL IgG positiva & 0 & $1(1,2 \%)$ & $1,000 *$ \\
\hline aCL IgM positiva & $4(4,9 \%)$ & $3(3,7 \%)$ & $1,000^{*}$ \\
\hline aCL IgA positiva & $1(1,2 \%)$ & 0 & $1,000^{*}$ \\
\hline antibeta2-gpl & $2(2,4 \%)$ & $4(4,9 \%)$ & $0,682^{*}$ \\
\hline $\begin{array}{l}\text { lgG positiva } \\
\text { antibeta2-gpl }\end{array}$ & $12(14,6 \%)$ & $8(9,8 \%)$ & $0,340 * *$ \\
\hline IgM positiva & & & \\
\hline $\begin{array}{l}\text { antibeta2-gp| } \\
\text { IgA positiva }\end{array}$ & $22(26,8 \%)$ & $12(14,6 \%)$ & $0,054^{* *}$ \\
\hline
\end{tabular}

\begin{tabular}{|c|c|c|c|}
\hline \multicolumn{4}{|c|}{$\begin{array}{l}\text { Tabela IV - Odds ratio para anticorpos anticardiolipina e antibeta2-gpI } \\
\text { ajustados para fatores de risco }\end{array}$} \\
\hline & $\mathrm{OR}^{\#}$ & IC95\%\#\# & $P^{*}$ \\
\hline $\mathrm{aCL} \lg \mathrm{G}^{* *}$ & não calculado & --- & -.- \\
\hline $\mathrm{aCL} \lg \mathrm{M}$ & 2,0 & $0,2-21,7$ & 0,570 \\
\hline $\mathrm{aCL} \lg A^{* *}$ & não calculado & --. & -.. \\
\hline antibeta2-gpl lgG & 0,1 & $0-1,0$ & 0,055 \\
\hline antibeta2-gpl IgM & 1,2 & $0,4-4,0$ & 0,726 \\
\hline antibeta2-gpl lgA & 3,4 & $1,3-9,1$ & 0,015 \\
\hline \multicolumn{4}{|c|}{$\begin{array}{l}\text { \#OR: odds ratio ajustado para dados demográfioc e fatores de risco; } \\
\text { \#\#IC95\%: intervalo de confiança de } 95 \% \text {; *teste de Wald }{ }^{17} \text {; **regressão } \\
\text { logística não calculada devido à freqüência nula em casos ou controles. } \\
\text { OR não ajustado após correção de Agresti }{ }^{18} \text { para teste IgG aCL foi } 0,3 \text { e } \\
\text { para IgA aCL foi } 3,0 \text {. }\end{array}$} \\
\hline
\end{tabular}

e cardiopatia prévia compreenderam as associações mais consistentes com infarto agudo do miocárdio.

Nossos resultados indicaram uma freqüência nula de anticorpos IgG anticardiolipina em casos de infarto. O OR não ajustado $(0,3)$ sugeriu um papel protetor para este isotipo, mas o dado é somente hipotético ( $P=1,000)$. Nosso grupo já havia relatado uma prevalência muito baixa (1.2\%) de IgG anticardiolipina em infarto agudo do miocárdio ${ }^{19}$. Entretanto, a presença de anticardiolipina IgG foi ligada a risco, embora pequeno, de infarto de acordo com relato anterior ${ }^{20}$. Duas coortes prévias relataram uma associação tempo-dependente de anticorpos IgG anticardiolipina com infarto agudo do miocárdio ${ }^{21,22}$.
Nossos dados em relação ao isotipo anticardiolipina lgM não foram compatíveis com associação com infarto agudo do miocárdio. A prevalência de anticardiolipina IgM em nosso estudo de 1993 foi nula ${ }^{19}$. Um recente estudo associando IgM anticardiolipina a acidente vascular cerebral traz à discussão o papel de infecções na resposta antifosfolipidica lgM ${ }^{23}$.

A freqüência de anticardiolipina lgA em nossos casos com infarto agudo do miocárdio foi bastante baixa (1,2\%). Nenhum controle foi positivo. O OR não-ajustado de 3,0 pode sugerir uma associação com o desfecho isquêmico. Entretanto, o valor $P$ nãoajustado de 1,000 torna esta hipótese improvável. Uma associação prospectiva do isotipo IgA anticardiolipina com infarto agudo do miocárdio foi previamente descrita ${ }^{21}$. Desta forma, o isotipo IgA aCL IgA, cujo imunoensaio ainda não é internacionalmente padronizado, deve ser melhor estudado nesses pacientes.

A relação de beta2-gp I com aterosclerose é intrigante. Ateromas contêm beta2-gpl ${ }^{6}$. Nosso estudo levanta a possibilidade de que anticorpos antibeta2-gpl estejam associados a risco de infarto agudo do miocárdio.

Em nosso estudo, a freqüência de anticorpos IgG antibeta2gpl foi menor em casos do que em controles. O baixo OR ajustado $(0,1)$ e o valor $\mathrm{P}$ ajustado de 0,055 trazem à tona a possibilidade de um papel protetor para este anticorpo.

Farsi e cols. ${ }^{11}$ relataram uma associação de anticorpos IgG antibeta2-gpl IgG com aterosclerose coronariana (particularmente, angina instável). No entanto, os dados do Honolulu Heart Program ${ }^{20}$ apontaram para uma freqüência insignificante de anticorpos IgG antibeta2-gpl IgG comparada a controles. Dois outros estudos também afastaram a possibilidade de anticorpos IgG antibeta2-gpl estarem ligados à doença coronariana ${ }^{3,24}$. Além disto, a presença de IgG antibeta2-gpl, bem como de anticorpos anticardiolipina, não pareceu conferir risco de reestenose coronariana após angioplastia ${ }^{25}$.

Nossos achados no isotipo IgM antibeta2-gpl não indicaram associação com infarto agudo do miocárdio. Em teoria, uma eventual resposta IgM antibeta2-gpl vista no infarto poderia resultar de infecção ou necrose tecidual prévia.

Anticorpos IgA antibeta2-gpl foram, significantemente, detectados em pacientes com infarto agudo do miocárdio quando com- 
parados a controles. O OR e o valor $\mathrm{P}$ ajustados indicaram que um teste positivo para IgA antibeta2-gpl se comportou como um fator de risco independente para infarto agudo do miocárdio. De forma similar, uma associação entre este anticorpo e risco de infarto cerebral foi recentemente relatada por nosso grupo ${ }^{26}$.

A associação de anticorpos IgA antibeta2-gpl com infarto agudo do miocárdio traz consigo algumas controvérsias. A grande maioria dos nossos pacientes com anticorpos IgA antibeta2-gpl é IgA aCLnegativa. Como previamente sugerido, é provável que IgA antibeta2gpl e IgA aCL compreendam AAF de diferentes especificidades ${ }^{27}$.

Se pacientes com infarto agudo do miocárdio IgA antibeta2gpl positivos mas com teste negativo para IgA aCL deveriam ser manejados como tendo síndrome antifosfolipídica, é motivo de debate. O consenso de 1999 para diagnóstico de síndrome antifosfolipídica não inclui anticorpos antibeta2-gpl ${ }^{2}$. A incorporação destes anticorpos aos critérios de síndrome antifosfolipídica foi proposta recentemente ${ }^{28}$.

De interesse, anticorpos contra o cofator fosfolipídico protrombina também estão implicados como fatores de risco para infarto agudo do miocárdio em homens de meia idade, de acordo com um relato ${ }^{29}$. Baixos níveis de anexina $\mathrm{V}$, um cofator fosfolipídico com propriedades anticoagulantes, foram recentemente descritos em pacientes com história de infarto agudo do miocárdio precoce ${ }^{30}$.

Em suma, anticorpos IgA antibeta2-gpl pareceram se comportar como fatores de risco independentes para infarto agudo do miocárdio em nosso estudo. A necessidade de se testar anticorpos antibeta2-gpl, particularmente IgA, em pacientes com doença coronariana cardíaca deve ser discutida e o valor preditivo avaliado.

Embora beta2-gpl seja encontrada em placas ateroscleróticas ${ }^{6}$, um papel patogênico para anticorpos IgA antibeta2-gpl no infarto agudo do miocárdio ainda não foi comprovado. Epifenômeno ou não, a ocorrência destes anticorpos no infarto agudo do miocárdio pode representar um dos elos entre autoimunidade e aterosclerose coronariana. As implicações clínicas de tais achados provavelmente serão esclarecidas em um futuro próximo.

\section{Agradecimentos}

A Vicki J. Nelson pela assistência técnica e aos Fundos Remanescentes da Sociedade Brasileira de Reumatologia.

\section{Referências}

1. Wick G, Xu Q. Atherosclerosis: an autoimmune disease. Exp Gerontol 1999; 34: 559-66.

2. Wilson WA, Gharavi AE, Koike T et al. International consensus statement on preliminary classification criteria for definite antiphospholipid syndrome. Arthritis Rheumatism 1999; 42: 1309-11.

3. Vaarala O. Antiphospholipid antibodies and myocardial infarction. Lupus 1998; S2 132-4.

4. Cervera R, Piette JC, Font J et al. Antiphospholipid syndrome: clinical and immunologic manifestation and patterns of disease expression in a cohort of 1,000 patients. Arthritis Rheum 2002; 46: 1019-27.

5. Kandiah DA \& Krilis S. Beta2-gpl. Lupus 1994; 3: 207-12.

6. George J, Harats D, Gilburd B, Afek A, Levy Y, Schneiderman J, Barshack I, Kopolovik J \& Shoenfeld Y. Immunolocalization of beta2-gpl (apolipoprotein H) to human atherosclerotic plaques: potential implications for lesion progression. Circulation 1999; 99: 2227-30.

7. George J, Afek A, Gilburd B et al. Induction of early atherosclerosis in LDL-receptor-deficient mice immunized with beta2-gpl. Circulation 1998; 98: 1108-15.

8. Arvieux J, Roussel \& Colomb MG (1994). Anticorps antiphospholipids et antibeta2-gpl. Ann Biol Clin 52: 381-5.

9. Martinuzzo ME, Pombo G, Forastiero RR et al. Lupus anticoagulant, high levels of anticardiolipin and anti-beta2-gpl antibodies are associated with chronic thromboembolic pulmonary hypertension. J Rheumatol 1998; 25: 1313-9.

10. Chen WH, Liu JS. An unusual increase of blood anti-beta2-gpl antibody but not antiphospholipid antibody in cerebral ischemia: a case report. Angiology 2001; 52: 149-54.

11. Farsi A, Domeneghetti MP, Fedi S et al. High prevalence of anti-beta2-gpl antibodies in patients with ischemic heart disease. Autoimmunity 1999; 30: 93-8.

12. Seventh report of the Joint National Commitee on the Prevention, Detection, Evaluation, and Treatment of High Blood Pressure (JNC 7): resetting the hypertension sails. Hypertension 2003; 41: 1178-9.

13. Donahue RP, Abbott RD, Reed DM, Yano K. Physical activity and coronary heart disease in middle-aged and elderly men. The Honolulu Heart Program. Am J Public Health 1988; 78: 683-5.

14. Gharavi AE, Harris EN, Asherson RA \& Hughes GRV (1987). Antiphospholipid antibodies: isotype distribution and phospholipid specificity. Ann Rheum Dis 1987; 46: 1-6.

15. Lewis S, Keil LB, Binder WL \& DeBari VA (1998). Standardized measurement of major immunoglobulin class (IgG, IgA, IgM) antibodies to beta2-gpl in patients with antiphospholipid syndrome. J Clin Lab Anal 1998; 12: 293-7.
16. Hopkins WG: A new view of statistics. On line. Available: http://www.sportsci.org/ resource/stats/index. Captured in April $11^{\text {th }}, 2002$.

17. Hosmer DW, Lemeshow S. Applied logistic regression. New York, NY: John Wiley \& Sons Inc; 1989.

18. Agresti A. Categorical data analysis. New York, NY: Wiley;1990.

19. Calcagnotto ME, Vitola D, Rabin M et al. Anticardiolipin antibodies and myocardial infarction. Rev Bras Reumatol 1993; 33: 217-20.

20. Brey RL, Abbott RS, Curb D et al. Beta2-gpl dependent anticardiolipin antibodies and risk of ischemic stroke and myocardial infarction. The Honolulu Heart Program. Stroke 2001; 32: 1701-6.

21. Vaarala $\mathrm{O}$, Manttari M, Manninem $\mathrm{V}$ et al. Anticardiolipin antibodies and risk of myocardial infarction in a prospective cohort of middle-aged men. Circulation 1995; 91: 23-7.

22. Wu R, Nityanand S., Berglund L, Lithell H, Holm G, Lefvert AK. Antibodies against cardiolipin and oxidatively modified LDL in 50-year-old men predict myocardial infarction. Arterioscler Thromb Vasc Biol 1997; 17: 3159-63.

23. Tuhrim S, Rand JH, Wu X et al. Elevated anticardiolipin antibody titer is a stroke risk factor in a multiethnic population independent of isotype or degree of positivity. Stroke 1999; 30: 1561-5.

24. Limaye V, Beltrame J, Cook R, Gillis D, Pile K. Evaluation of antibodies to beta2$\mathrm{gpl}$ in the causation of coronary atherosclerosis as part of the antiphospholipid syndrome. Aust NZ J Med 1999; 29: 789-93.

25. George J, Harats D, Bakshi E et al. Anti-oxidized low density lipoprotein antibody determination as a predictor of reestenosis following percutaneous transluminal coronary angioplasty. Immunol Lett 1999; 68: 263-6.

26. Staub HL, Norman GL, Crowther T et al. Antibodies to the atherosclerotic plaque components beta2-glycoprotein I and heat-shock proteins as risk factors for acute cerebral ischemia. Arq Neuropsiquiatr 2003; 61: 757-63

27. Tsutsumi A, Matsuura E, Ichikawa K et al. Antibodies to beta2-gpl and clinical manifestations in patients with systemic lupus erythematosus. Arthritis Rheum 1996; 39: 1466-74.

28. Piette JC. Towards improved criteria for the antiphospholipid syndrome. Lupus 1998; 7 Suppl 2: S149-57.

29. Vaarala 0 . Antibodies to prothrombin imply a risk of myocardial infarction in middle-aged men. Thromb Haemost 1996; 75: 456-9.

30. Roldán V, Marin F, Pineda J et al. Anexin V levels in survivors of early myocardial infarction. Rev Esp Cardiol 2002; 55: 1230-4. 